\title{
Dynamic Resource Management in MC-CDMA Based Cellular Wireless Networks
}

\author{
$\underline{\text { doi:10.3991/ijim.v3s2.890 }}$ \\ Dhananjay Kumar, C. Chellappan, B. Bala Jeevitha Vani \\ Department of Information Technology, Anna University, Chennai, India
}

\begin{abstract}
Most of the multimedia and Internet services today are asymmetric in nature and require high data rate support. Allocating equal band width in both uplink and downlink is not a prudent solution, as most of the time user requirements are either greater in uplink or downlink. The Multi Carrier Code Division Multiple Access (MC-CDMA) system with Time Division Duplex (TDD) mode can easily meet this requirement by dynamically declaring traffic direction in the TDD slot and adaptively allocating the sub channels. In this paper, we propose an adaptive slot and sub carrier allocation algorithm that can be independently implemented in each cell of a mobile communication network. Our analytical model is the generalization of two cell concept to represent a multi cell model. Based on the two cell concept, four cases of interference pattern have been considered and simulated separately in presence of Additive White Gaussian Noise (AWGN) and Rayleigh Channel. The simulated result suggests the requirement of approximately 9dB of Signal to Noise Ratio (SNR) to maintain Bit Error Rate (BER) below $10^{-3}$. We also analyze the average delay incurred by the proposed algorithm in allocating resources.
\end{abstract}

Index Terms-BER, TDD, MC-CDMA, SNR, Delay

\section{INTRODUCTION}

The explosive growth in multimedia and Internet applications necessitates the development of next generation mobile networks. The multi-carrier communication systems, because of their inherent property, have become a natural choice for Fourth Generation (4G) systems [12]. The conventional code-division multiple-access (CDMA) technique used in third generation system faces serious limitations by fading problem in channel, causing Inter Symbol Interference (ISI), and it requires advanced signal processing algorithms to contain it [3]. The MCCDMA employing multiple stream of data channel can combat channel dispersion, hence ISI, thereby increasing system capability to accommodate higher number of users and different multimedia services.

The efficiency in the multiple access techniques becomes an important issue as the demand for high data rate to support Internet applications continue to grow. Many applications demand more bandwidth either in forward channel or in reverse channel, and popular applications like web browsing are biased towards downlink. Allocating equal resources in both uplink and downlink becomes a bottleneck for the system as uplink remains underutilized while downlink gets strained. The TDD mode not only allows efficient utilization of spectrum but also flexible resource allocation that can easily support this asymmetric traffic. Further the MC-CDMA shows higher efficiency by adopting adaptive modulation techniques like M-ary Quadrature Amplitude Modulation (M-QAM) [4-5]. By dynamically allocating subcarriers and an adaptive slot management the system can meet the large dynamic resource requirements of a real-time multimedia application in the Internet.

Due to multiple reflections from various objects, the electromagnetic waves reach the receiver along different paths of varying lengths. The interaction between these waves causes multipath fading at a specific location, and also decreases the strength of the radio wave with respect to the increase in distance from transmitter. Even when line-of-sight exists, random phenomenon, such as, reflection, refraction, diffraction, and scattering weaken the signal and result in multiple reception of the signal with different delays and strength (Fig.1). When the mobile station is stationary it may receive signals with constant strength, but when it moves the received signal fluctuates. In other words, due to multipath phenomenon the channel frequency response becomes irregular as shown in Fig.2

Single carrier systems are simple but can't support high data rates efficiently in fading channels. The multi carrier system can not only improve performance in bad channel conditions, but also support high data rates while maximizing the capacity of the system. The other advantages of multi carrier system include flexible resource allocation to support different bit rate services.

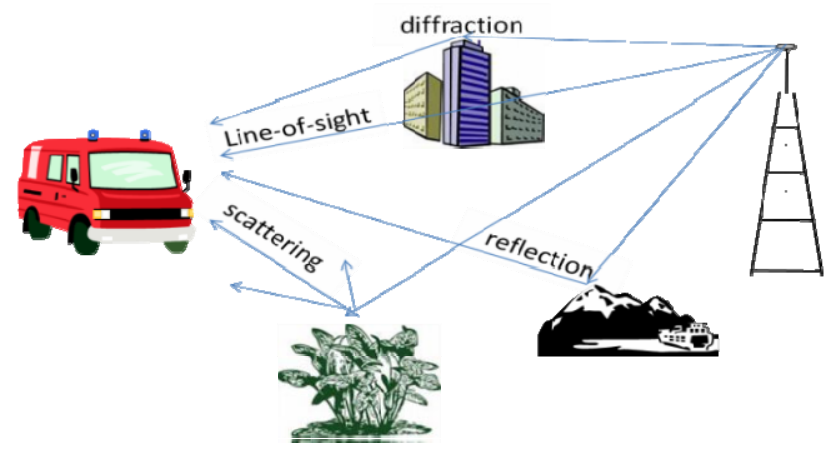

Figure 1. Multipath propagation

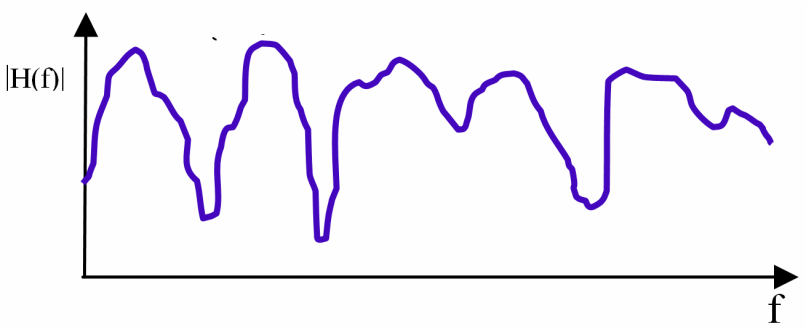

Figure 2. Multipath channel frequency response 


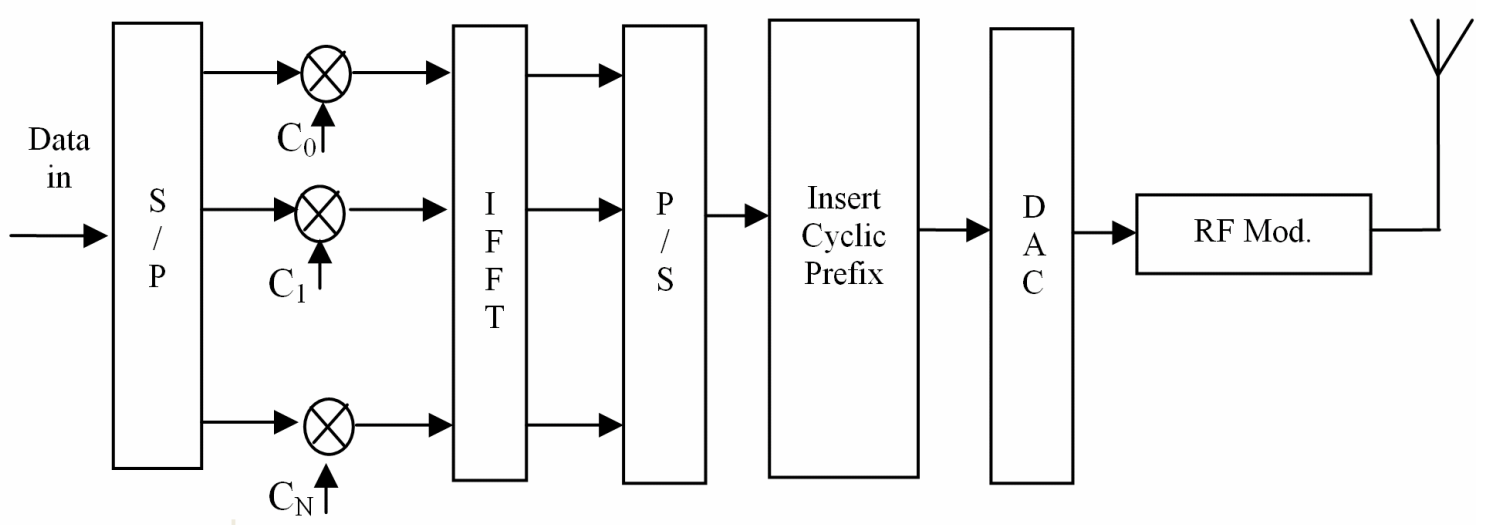

Figure 3. MC-CDMA Transmitter

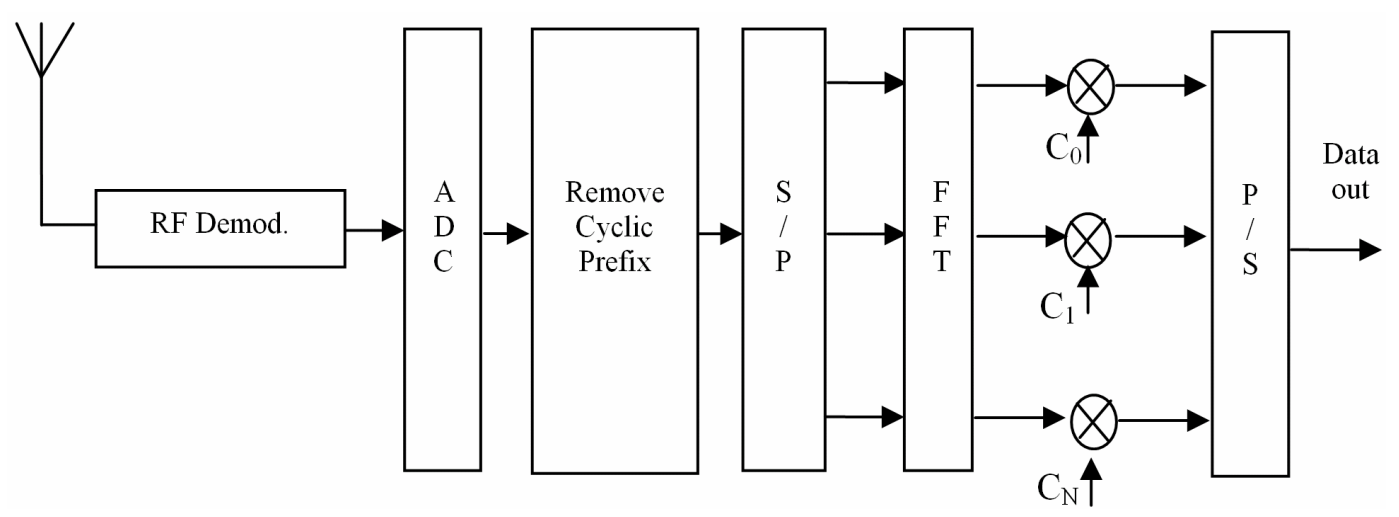

Figure 4. MC-CDMA Receiver

The CDMA based systems are inherently interference limited. The presence of every user affects the SNR, and hence data supported on a particular channel. The channel state information [6-7] can be estimated periodically with the help of a pilot carrier, and based on the estimated value the order of modulation $\mathrm{M}$ can be decided, thus optimizing the system capacity.

A simplified block diagram of MC-CDMA is presented in Fig. 3 \& 4. The serial input data is converted to parallel before spreading by a CDMA code. Then the spread data is modulated using IFFT. The output of IFFT is converted back to the serial to be appended by a cyclic prefix. The cyclic prefix is added to mitigate the inter symbol interference. The Digital to Analog Converter (DAC) converts the cyclic prefix appended input data into analog, which further gets modulated by RF modulator. The reverse procedure is carried out at the receiver to decode the signal as shown in Fig.4.

Considering two channel: Pilot and data channel, the MC-CDMA M-QAM $(\mathrm{M}=2)$ signal transmitted by the $\mathrm{k}^{\text {th }}$ user can be given by[8],

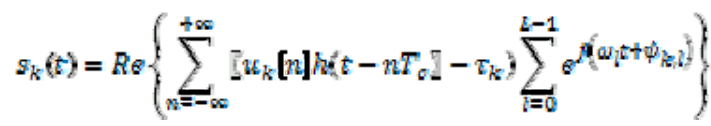

where

$$
u_{k}[\mathrm{~b}]=A C_{p k}[\mathrm{~b}]+B C_{d k}[\mathrm{n}] \mathbf{d} d_{k}[\mathrm{~b}]
$$

Here A \& B are the signal amplitude of the pilot and data channel respectively. $d_{k}[n]$ is the user data, $h(t)$ is the impulse response of the chip wave shaping filter, $\omega_{l}$ and

$k_{k_{t}}$ l are the carrier frequency and carrier phase of the $1^{\text {th }}$ subcarrier respectively and $\mathrm{L}$ is the no. of subcarriers.

Assuming that each sub channel experiences an independent flat Rayleigh fading, and a perfect average power control is employed, the received signal can be given by

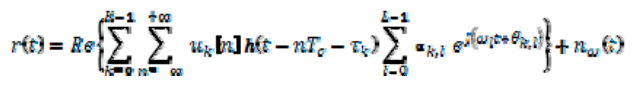

where, $\mathrm{K}$ is the total no. of users. $\tau_{k}$ is the relative time delay of user k. $\boldsymbol{\alpha}_{k, l}$ and $\theta_{k, l}$ are the fading amplitude $\&$ phase respectively of the $1^{\text {th }}$ path for the $\mathrm{k}^{\text {th }}$ user and $n_{w}(t)$ is zero mean white Gaussian noise with two sided spectral density $\$ / 2$.

In this paper the proposed algorithm tries to optimize the number of subcarrier and slot utilization for $\mathrm{MC}$ CDMA employing TDD technique. Every cell can have its own slot allocation method based on the traffic load. The SNR depends mainly on the direction of traffic (uplink / downlink) in home and interfering cells. In multicell environment, based on prevailing conditions many cases of interference pattern may arise. The system representation could be the generalization of two cell model for multi-cell. Further, the analysis presented here includes worst case scenario to evaluate system performance. The simulation carried out is the extension of the 
two cell model to represent MC-CDMA in time duplex mode.

The remaining part of this paper is organized as follows. In Section2, a comprehensive literature survey is presented. System model plays a vital role in defining the objective and conducting simulation. Section 3 represents multi-cell as well as two cell model for SNR estimation. The development of the proposed protocol is discussed in section4. The simulated result and analysis are shown in section5. Finally this paper concludes in section6.

\section{RELATED WORK}

The performance of adaptive resource allocation algorithm for MC-CDMA has been studied by many researcher under different scenario $[5,7,9]$. Mamoun Guenach, and Heidi Steendam [3] investigated the sensitivity of downlink MC-CDMA performance considering the optimum number of carriers and guard interval. They derived SNR and tried to show that the load of the MC-CDMA system only has small influence on the optimization of the parameters. U. O. Ibom [7] has proposed an adaptive MC-CDMA for next generation network. He has observed the effect of bit allocation \& BER in presence of white noise, and a noise with exponential spectrum. The performance study of MC-CDMA in crosslayer resource allocation by Virginia Corvino et al.[9] demonstrates user outage rate depending on scheduling and traffic load.

In [10-11], BER performance evaluation under different channel model has been carried out. Çetin Kurnaz and Hulya Gokalp [10] have investigated the performance of the downlink in MC-CDMA systems using a channel model from in-the-field propagation measurements. They have studied the effect of transmission bandwidth, number of users and number of sub-carriers on system performance. Throughput was the focus of attention in observation made by L. Guerrero et al. [11] in Nakagami-m fading channels.

The BER Comparison of OFDM and MC-CDMA system has been carried out by [12] Muhammad Talha Ahmed et al. [12]. Their observation in Rayleigh fading channel in presence of additive white Gaussian noise shows that MC-CDMA outperforms OFDMA. S. Chatterjee et al.[4] have analyzed BER performance of adaptive modulation based MC-CDMA systems for $4 \mathrm{G}$ wireless systems. They have considered different modulation schemes for simulation.

Although many research papers have been presented on performance of MC-CDMA, the effect of dynamic slot management using adaptive modulation in time division duplex mode has not been studied. This paper presents an algorithm that optimally manages the resources to maximize the system performance.

\section{SYSTEM MODEL}

In MC-CDMA because of traffic asymmetry and hence different slot allocation from cell to cell, the system suffers from both intra-cell and inter-cell interference (Fig.5). For example, mobiles in cell1 may use uplink slots and at the same time the base station of an adjacent cell ie.cell2 may use downlink slot to transmit signals. In this situation, the uplink (downlink) channel in a cell can be interfered by the downlink (uplink) of the adjacent cell and, in turn, this results in capacity degradation.

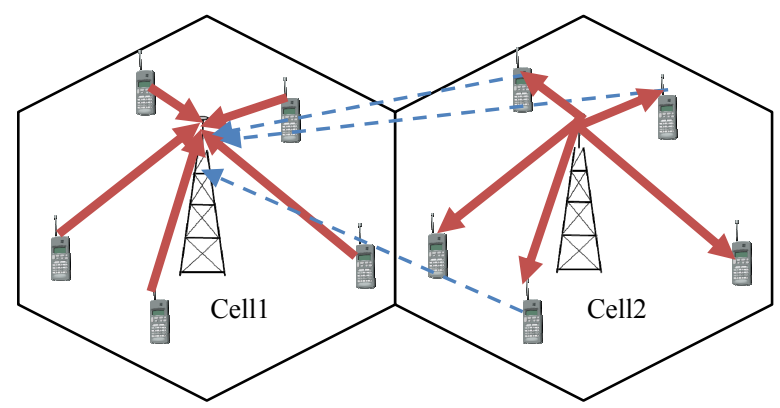

Figure 5. Interference in uplink and downlink because of cross-slot

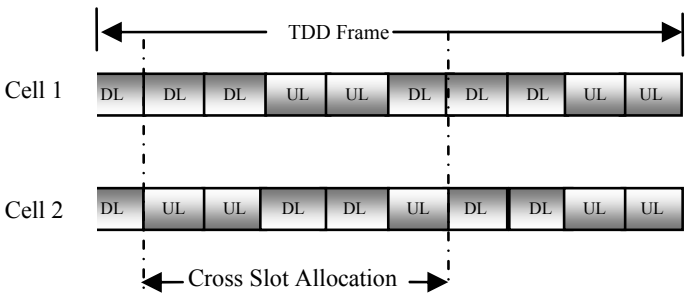

Figure 6. Different slot allocation in a adjacent cell

Although a different slot allocation within a cell is also possible, practically it is not implemented as it will cause heavy interference in both uplink and downlink. Here, we assume that slot allocation within a cell is same and perfectly synchronized between base station (BS) and mobile station (MS). Cross slot allocation between two cells is the case for traffic asymmetry (Fig.6). The number of cross slot allocations can be governed by the base station controller (BSC) in a location area consisting of multiple cells. Since cross slot allocation normally causes more interference, it will be a major factor in supporting quality of service and also capacity of the system.

\section{A. SNR in Multi-cell TDD Environment}

In MC-CDMA with TDD, each slot will carry many user data on different channels separated / identified by Walsh-Hadamard code. Considering intra-cell and intercell interference separately in a multi-cell environment, the signal to noise ratio for each user's $i^{\text {th }}$ subcarrier can be modeled as

$$
S W R_{i}=\frac{R_{p} \cdot S F}{I_{m e}+Z_{\text {ext }}+N_{\mathrm{Q}} W}
$$

Where $\mathrm{P}_{\mathrm{r}}$ is the received power, SF is the spreading factor, $I_{\text {int }}$ is the internal noise within the cell, $I_{\text {ext }}$ is external noise coming from other cells, $\mathrm{N}_{\mathrm{o}}$ is the noise power spectral density, and $\mathrm{W}$ is the total transmission bandwidth.

To approximate link gain, the received power $\mathrm{P}_{\mathrm{r}}$ at BS, can be related to the transmit power $\mathrm{P}_{\mathrm{t}}$ of the MS as

$$
R_{p}=\lambda d^{-v} R_{t}
$$

Where $\mathrm{d}$ is the distance between BS and MS and $\lambda$ is a constant. In mobile communication, the loss of power in propagation is inversely proportional to the $4^{\text {th }}$ power (i.e. $\mathrm{v}=4$ ) of the distance between transmitter and receiver [14]. 
In MC-CDMA a channel is represented by a no. of sub-channels/carriers. The no. of subcarriers needed to support an application is dictated mainly by the bandwidth requirement. Further, the sub-carrier selection is based on their current SNR.

System model for MC-CDMA in multi-cell environment can be built by generalizing the two cell models. Considering the two cell approach, four cases arise: i. cell1 uplink cell2 uplink, ii. Cell1 uplink cell2 downlink, iii. Cell1 downlink cell2 uplink, and iv. Cell1 downlink cell2 downlink. Here, cell1 represents a home cell for tagged mobile and cell 2 represents a cell in first tier interfering cells.

\section{1) Cell1 Uplink Cell2 Downlink}

Suppose $m_{k}$ is the number of MS served by a channel, where $\mathrm{k}=1,2,3, \ldots \ldots \mathrm{K}$. Let $\mathrm{P}_{\mathrm{k}}^{\mathrm{i}}$ denote the transmit power of $\mathrm{i}^{\text {th }}$ subcarrier, and $\mathrm{H}_{\mathrm{k}}^{\mathrm{i}}$ the channel gain between $\mathrm{i}^{\text {th }}$ MS and its BS. The internal interference $\mathrm{I}_{\text {int }}$ in uplink for $\mathrm{i}^{\text {th }}$ subcarrier carrying part of $\mathrm{k}^{\text {th }} \mathrm{MS}$ data may be given by

$$
I_{\text {fnt }}=\sum_{k=1}^{m_{k}} \sum_{1-1}^{l_{k}} H_{h}^{L} \cdot P_{k}^{L}
$$

The external interference $I_{e x t}$ is computed as follows. The BS present in neighboring cell $\mathrm{J}$ will cause interference to the uplink signal of tagged mobile. Now the $I_{\text {ext }}$ can be represented as

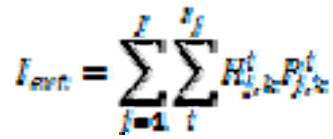

\section{2) Cell1 Downlink Cell2 Uplink}

Most commonly data in downlink channels (for example in W-CDMA) are transmitted with orthogonal codes. Assuming perfect time synchronization between MS and $\mathrm{BS}$, and if the channel is of flat fading type, then orthogonality is preserved during downlink slot and hence the internal noise $I_{\text {int }}$ is absent. But the multipath propagation damages the orthogonality. An orthogonality factor $(\alpha)$, which is the percentage of downlink orthogonality remaining at the mobile receiver, is introduced to compute $I_{\text {int }} \alpha$ is 1 for a signal without multipath, and near zero in Rayleigh fading environment.

The internal interference $I_{\text {int }}$ arising due to nonorthogonality of the received signals is given by

$$
E_{t n t}=\sum_{i=1}^{i} \alpha_{t} H_{t} P_{t}
$$

where, $\mathrm{P}_{\mathrm{i}}$ is the transmit power of the $\mathrm{BS}, \mathrm{H}_{\mathrm{i}}$ the channel gain, and $\alpha_{i}$ the corresponding orthogonality factor for the $\mathrm{i}^{\text {th }}$ subcarrier.

To compute external interference $\mathrm{I}_{\mathrm{ext}}$, it is assumed that same sub-carriers are used in neighboring cell. Let $m_{j}$ be the number of MS served in a $\mathrm{j}^{\text {th }}$ cell, where $\mathrm{j}=1,2, \ldots \ldots \mathrm{J}$ represents $\mathrm{J}$ neighbor cells. Now $\mathrm{I}_{\text {ext }}$ may be expressed as

$$
\mathrm{I}_{\mathrm{ext}}=\sum_{\mathrm{j}=1}^{\mathrm{J}} \sum_{\mathrm{h}=1}^{\mathrm{m}_{1}} \sum_{\mathrm{i}}^{\mathrm{I}_{\mathrm{h}}} \mathrm{H}_{j \mathrm{~h}}^{\mathrm{i}} \mathrm{P}_{\mathrm{ih}}^{\mathrm{i}}
$$

where $\mathrm{H}_{\mathrm{j}, \mathrm{k}}^{\mathrm{i}}$ the link gain between $\mathrm{i}^{\text {th }}$ MS in neighbor cell and the tagged $B S$, and $\mathrm{P}_{\mathrm{j}, \mathrm{k}}$ the transmit power of $\mathrm{i}^{\text {th }}$ MS to support its QoS requirement in its cell.

\section{3) Cell1 Uplink Cell2 Uplink}

In this case, the $\mathrm{I}_{\text {int }}$ is same as in section 3.1.1 given by (6). The external interference $\mathrm{I}_{\mathrm{ext}}$, is given by (9).

\section{4) Cell1 Downlink Cell2 Downlink}

Here the internal interference $I_{\text {int }}$ and external interference $I_{\text {ext }}$ are given by (8) and (7) respectively.

\section{B. Delay Estimation}

The total must be upper bounded as per the QoS requirement of real time services. Here, we first talk about different components of end to end delay, but our main focus is on processing delay due to resource allocation algorithm at base station. The major components of delay are discussed as follows [15].

1) Packetization Delay

The packetization delay is incurred by the Codec to encapsulate raw data into packets. It is given by

$$
T_{P D}=\frac{F_{Y}}{f_{P T r}} \quad \text { [ns] }
$$

where $F_{S}$ is the payload size and $\tau_{g W}$ is codec bandwidth in $\mathrm{kbits} / \mathrm{s}$.

2) Decompression delay

It is on an average $10 \%$ of the compression delay. So, the decompression delay can be defined as

$$
T_{D C D}=0,1 * N * T_{C D} \quad[\mathrm{~mm}]
$$

Where, $T_{D C D}$ decompression delay in $\mathrm{ms}, \mathrm{N}$ is is no. of the voice blocks in the packet, and $T_{C D}$ is coder delay in ms.

3) Delay in Transmission Network

This delay depends upon the link band width and payload and header size, and is given by

$$
T_{S E K}=\frac{R_{z}+H_{E}}{L_{g}} \quad[\mathrm{~m} m]
$$

where, $L_{2}$ is the line speed in $\mathrm{kbit} / \mathrm{s}$, and $A_{L}$ is the header length in bit.

4) Processing delay

This delay happens at the base station of the MCCDMA system. It has the following components: (i) serial to parallel conversion time, (ii) spreading delay i.e. application of modulo-2 operation on each bit, (iii) computation of IFFT, (iv) time spent on running resource allocation algorithm, and (v) delay incurred in cyclic prefix and DAC device. Almost the same amount of delay occurs in the receiver side except fourth component i.e. resource allocation. 


\section{Channel Model}

There are many models available in literatures to characterize a fading channel. Rayleigh channel model assumes a direct / dominant path and many reflected path. The probability density function of a Rayleigh fading channel is defined as follows [16].

$$
f_{\rho}(p)=f_{\rho}(\sqrt{2 \rho})\left|\frac{\alpha \rho}{\sigma_{p} p}\right|=\frac{1}{\sigma^{2}} \exp \left(\frac{-p}{\sigma^{2}}\right)
$$

where, $\mathrm{p}$ is instantaneous power and is related to signal amplitude $\rho$ as

$$
p=\frac{1}{2} \rho^{2}
$$

\section{Noise Model}

We consider an Additive White Gaussian Noise (AWGN) in presence of Rayleigh channel to simulate BER given by

$$
\sigma^{2}=\frac{W * N_{0}}{N}
$$

where $\mathrm{W}$ is the total bandwidth, $\mathrm{N}_{0}$ is the noise spectral density, $\mathrm{N}$ is total subcarriers.

\section{E. BER Calculation}

The BER for the $\mathrm{i}^{\text {th }}$ Sub-Carrier corresponding to MQAM is given by [18]

$$
B E R_{t}=\frac{1}{5} * \exp \left[\frac{\left(-1.5 * S N R_{t}\right)}{M-1}\right]
$$

where $\mathrm{SNR}_{\mathrm{i}}$ is the signal to noise ratio for $\mathrm{i}^{\text {th }}$ subcarrier, and $\mathrm{M}$ is the constellation points in M-QAM.

\section{SubCARrier AND Slot AllocAtion Algorithm}

The proposed algorithm manages the subcarrier and slots to meet the quality of service requirement of an application. The selection of subcarriers is carried out based on its current SNR to support a minimum BER. The slot management algorithm decides whether an outgoing slot is to be declared as uplink/downlink based on the existing capacity of the present slot. First, the SNR is calculated using the formulae shown in section 3, that falls under any one of the four cases considered there. Since the CDMA based systems are inherently interference limited, the SNR is recomputed every time based on the new call arrival rate, and hence the addition of a new subcarrier in a slot. The new call includes handoff user too. The BER is computed using (16) based on the SNR and a high modulation order $(\mathrm{M}=8)$. If BER $>10^{-3}$, then order of modulation $\mathrm{M}$ is reduced and BER is computed again, and this process is repeated till BER falls below $10^{-3}$ and the corresponding $\mathrm{M}$ value is retained to be used for order of modulation in M-QAM. If BER does not fall below $10^{-3}$ and $\mathrm{M}=2$, then the next slot is declared as same status (e.g. uplink if the current slot is uplink) and new calls are accommodated in new slots. Based on the existing SNR and application's bandwidth requirement, the no. of subcarriers are allocated to these new calls.

If the accumulated bandwidth $\left(\mathrm{BW}_{\mathrm{c}}\right)$ i.e. no of subcarriers are just enough to meet the requirements $\left(\mathrm{BW}_{\mathrm{r}}\right)$, the resource allocation completes for an user and the algorithm takes next call to be processed. Following are the steps of the proposed algorithm.

Step1: Pick up an unallocated sub carrier and compute the SNR

Step2: Estimate BER for the Modulation order (M)

Step3: If BER is below $10^{-3}$, retain the value of $\mathrm{M}$ and allocate this subcarrier. Else decrement $M$ by one and if $\mathrm{M}>2$ means go to Step2 .Otherwise declare next slot in same direction and allocate the subcarrier in that slot. Go to step1.

Step4: Check whether these allocated carrier meets the requirements.

Step5: If $\mathrm{BW}_{\mathrm{c}}>=\mathrm{BW}_{\mathrm{r}}$ means resource allocation for this particular user is over.

Step6: See if any more call is in waiting queue. If so, go to step1

Step7: If all calls are accommodated, the resource allocation procedure ends.

\section{RESULTS AND DISCUSSION}

Simulations were carried out for the four cases of uplink and downlink scenario of MC-CDMA system. First we simulated the BER performance of our proposed algorithm in presence of Rayleigh channel. Different Walsh codes were used for spreading user data on each subcarrier. Fig. 7 shows the traffic distribution in terms of subcarrier for different slots used in driving the simulation. The major simulation parameters are listed in table-I.

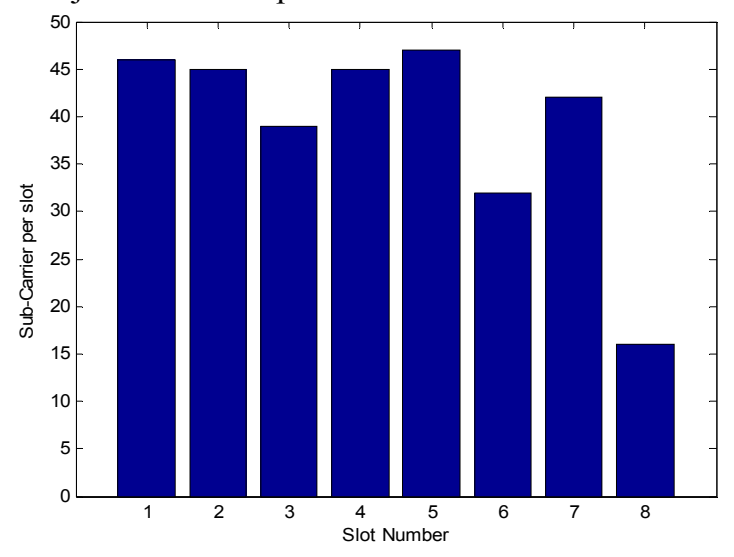

Figure 7. Input traffic distribution for simulation.

TABLE I.

SIMULATION PARAMETERS

\begin{tabular}{|l|l|}
\hline Bandwidth & $10 \mathrm{MHz}$ \\
\hline Spreading Factor & 128 \\
\hline FFT Length & 1024 \\
\hline Modulation (M-QAM) & $2-8$ \\
\hline Rayleigh Channel & $\mathrm{Ts}=0.01 \mathrm{~s}, \Delta \mathrm{f}=10 \mathrm{~Hz}$ \\
\hline Number of users & $15-30$ \\
\hline Length of Walsh Code & 8 \\
\hline
\end{tabular}




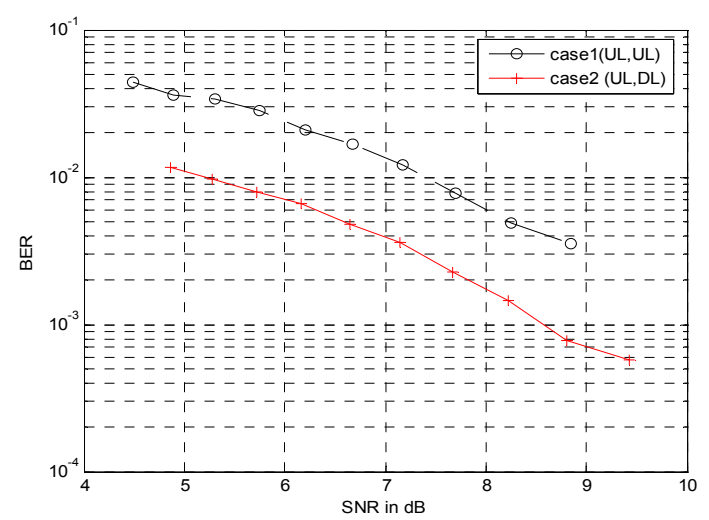

Figure 8. BER performance in case1 and case2.

TABLE II.

SIMULATION SCENARIO

\begin{tabular}{|l|l|l|}
\hline Case & Cell1 & Cell2 \\
\hline Case1 & Uplink (UL) & Uplink (UL) \\
\hline Case2 & Uplink (UL) & Downlink (DL) \\
\hline Case3 & Downlink (DL) & Downlink (DL) \\
\hline Case4 & Downlink (DL) & Uplink (UL) \\
\hline
\end{tabular}

The four cases considered here represent different scenarios as listed in Table-II. Each case represents a traffic direction, and the interference pattern changes accordingly. The BER in case 1 as shown in Fig. 8 follows a higher path because the uplink base station suffers from both intra cell and inter cell interference from a large no. of users.

As discussed earlier, in downlink the intra cell interference is caused by orthogonality factor. Fig.9 shows the BER performance with respect to variation in orthogonality factor. In case of cross slot, i.e. case 4 , there could be heavy interference, as interfering user in neighboring cell may be near to the tagged mobile. Case 3 shows a slight better performance, as interference is caused by a base station only as the neighboring cell is in downlink. A curve fitting function is used to plot BER here.

The distance ratio considered in Fig. 10 represents the ratio of distance between tagged mobile and base station to the mean distance of interfering mobile in the same cell. As indicated in (5), the received power inversely varies approximately with fourth power of distance in wireless communication, a small change in distance causes large changes in interference pattern and hence SNR.

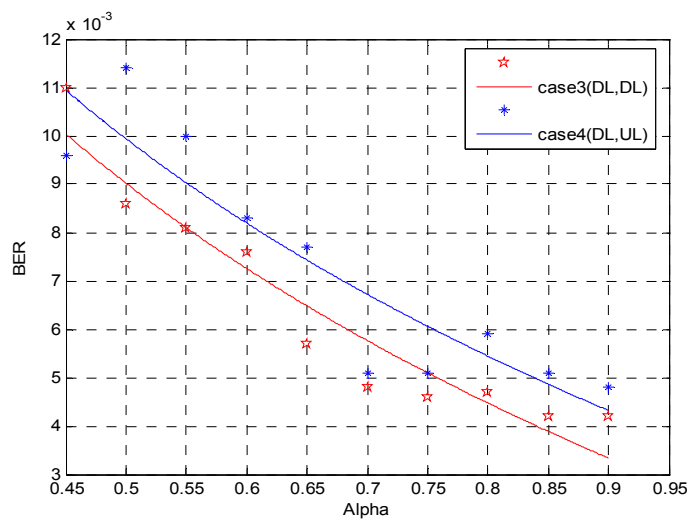

Figure 9. BER with respect to orthoganility factor in internal interference

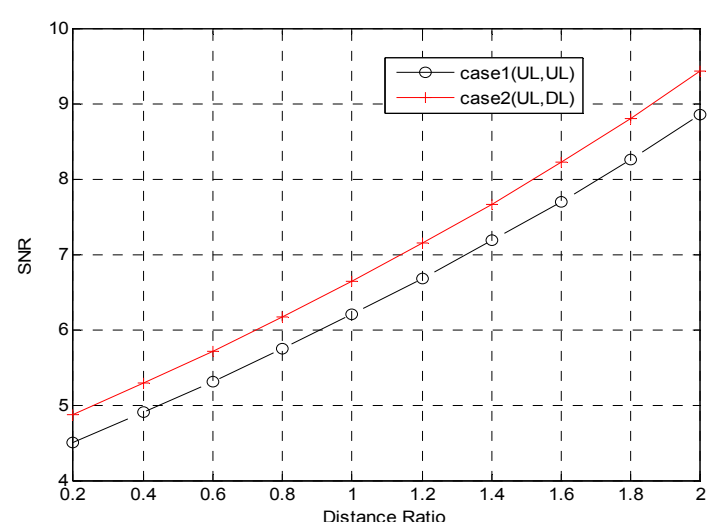

Figure 10. SNR vs distance ratio in internal interference

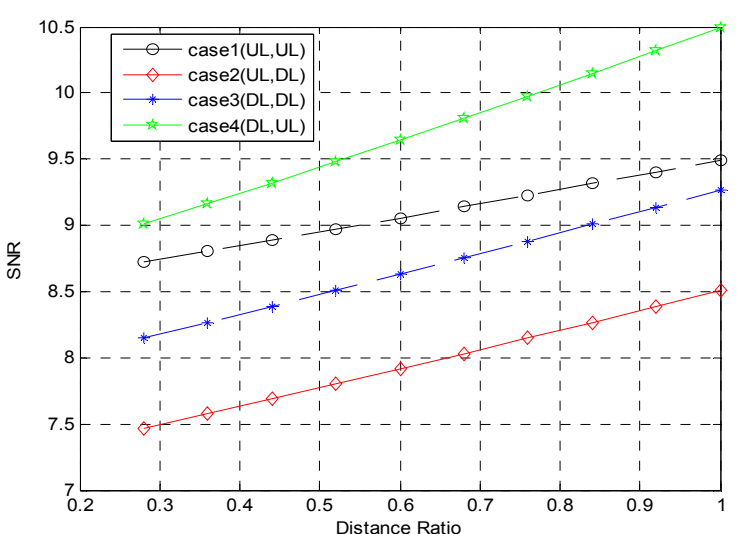

Figure 11. SNR with respect to distance ratio for external interference.

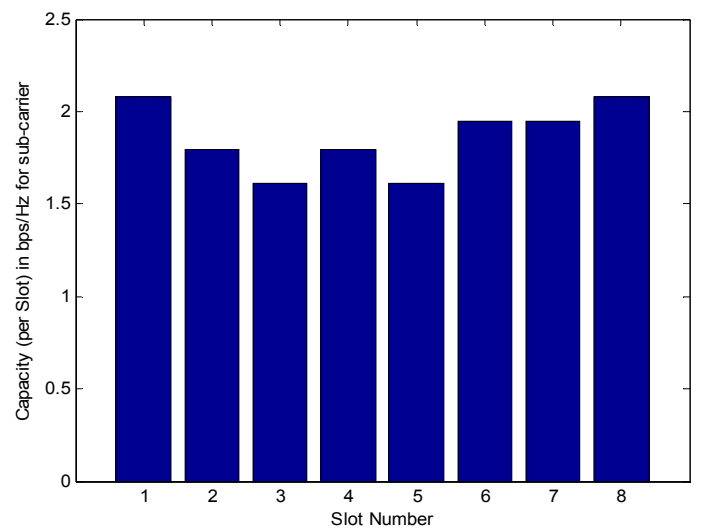

Figure 12. Capacity (b/s per Hz) per subcarrier

The capacity (b/s per Hz) of each subcarrier in a given slot is computed during simulation of the proposed algorithm and is plotted in Fig.12.. In the given scenario, since the maximum order of modulation is only eight, the achievable capacity will be $3 \mathrm{~b} / \mathrm{s}$ per $\mathrm{Hz}$ at most, as predicted by the Shannon's capacity formula.

Fig.13 shows the delay in processing new calls. The new calls may originate from a new user or due to a hand off call. The delay observed here is the total processing delay. Once the resource is allocated to a particular call, this delay will be constant for a particular service class. 


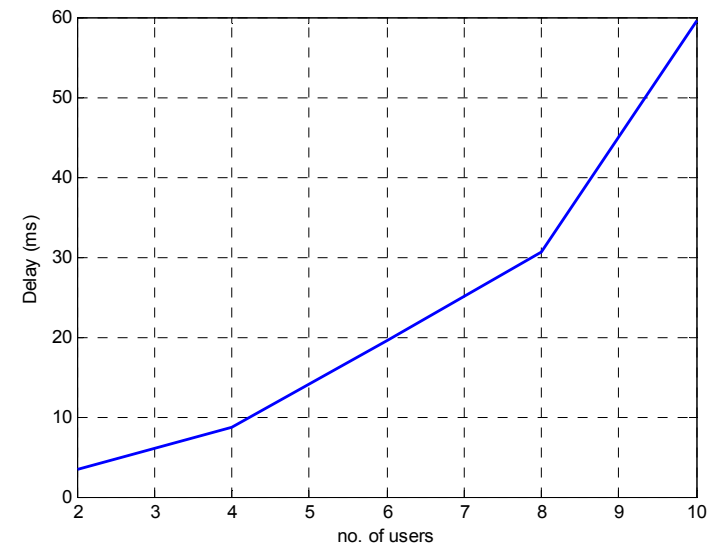

Figure 13. Delay with respect to no. of users

\section{CONCLUSION}

To analyze the performance of the proposed algorithm in MC-CDMA system the interference pattern corresponding to different scenario (four cases) was presented. The multi-cell environment was modeled and simulated by generalizing the two-cell model. The interference analysis has two major components: the intra cell and inter cell. The BER performance for different cases provides an overview of system analysis in presence of AWGN noise and Rayleigh channel. The capacity shown here is low because of using lower order of modulation. The delay analysis was carried out with respect to processing of new calls.

The system performance of MC-CDMA under TDD mode accounts for many parameters and it requires further analysis. The BER, delay and system capacity alone were analyzed here, and other system parameters like throughput need to be analyzed. Future work could also include the performance analysis of MC-CDMA based on efficient codes like orthogonal variable spreading factor code.

\section{REFERENCES}

[1] S. Plass, S. Kaisar, "MC-CDMA versus OFDMA in Cellular Environments", EUSIPCO 2005, Turkey, September 6, 2006.

[2] Filippo Giannetti, Vincenzo Lottici, and Ivan Stupia, "Iterative Multi-User Data Predistortion for MC-CDMA Communications", IEEE Transaction on wireless communication, Vol .7, No.10, October 2008.

[3] Mamoun Guenach, and Heidi Steendam, "Performance Evaluation and Parameter Optimization of MC-CDMA", IEEE Transactions on Vehicular Technology, vol. 56, no. 3, May 2007. (doi:10.1109/TVT.2007.895605)

[4] S. Chatterjee1, W.A.C. Fernando2, M.K. Wasantha1,"Adaptive Modulation based MC-CDMA Systems for 4G Wireless Consumer Applications", IEEE, June 2003.
[5] G.K.D. Prasanna Venkatesan, and V.C.Ravichandran, "Performance Analysis of Dynamic Sub-Carrier Allocation Technique for Adaptive Modulation based MC-CDMA System", IJCSNS International Journal of Computer Science and Network Security, VOL.7 No.2, February 2007.

[6] Uchennaya Obo Ibom, " Channel State Information Enhanced Adaptive MC-CDMA" Wireless Pervasive Computing, 2008. ISWPC 2008

[7] U. O. Ibom, “Adaptive MC-CDMA for NGN", IEEE ICMMT Proceedings, 2008

[8] Chong L.L, and Milstein L.B, "Error rate of a multicarrier CDMA system with imperfect channel estimates", 2000 IEEE International Conference on Communications (ICC 2000).

[9] Virginia Corvino, Velio Tralli, Roberto Verdone, "Cross-Layer Resource Allocation for MC-CDMA”, IEEE ISWCS 2007

[10] Cetin Kurnaz, Hulya Gokalp, "Investigating MC-CDMA System Performance Using Measurement-Based Channel Models", IEEE Xplore, 2008.

[11] L. Guerrero, F. Said, A. Lodhi, A.H. Aghvami, "Throughput of Distributed Cyclic Delay Diversity MC-CDMA Relaying Over Nakagami-M Fading Channels", IEEE WCNC 2008.

[12] Muhammad Talha Ahmed, Junaid Khalid, Khalida Noori, Sami Ahmed Haider , "Bit Error Rate Comparison of OFDM and MCCDMA Systems", International Conference on Computer Science and Information Technology, 2008.

[13] Klein S Gilhousen et. al., "On the Capacity of a Cellular CDMA System", IEEE Transaction on Vechicular Technology, Vol 40, No2, May1991.

[14] W. C.Y. Lee, Mobile Cellular Telecommunications, 2nd ed. NewYork: McGraw-Hill, 1995.

[15] Miroslav Vozňák - František, "Analytic model of a delay variation valid for the RTP”, Technical Report 16/2007. Available at http://homel.vsb.cz/ voz29/files/TR2007.pdf.

[16] JPL's Wireless Communication Reference Website http://www.wireless.per.nl/reference/chaptr03/ricepdf/ricepdf.htm

[17] T. S. Rappaport, "Wireless Communications Principles and Practice", Pearson Education, Inc., 2004.

[18] A. J. Goldsmith and S.-G. Chua, "Variable-rate variable-power MQAM for fading channels", IEEE Trans. Commun., vol. 45, pp. 1218-1230, Oct. 1997. (doi:10.1109/26.634685)

\section{AUTHORS}

Dhananjay Kumar is with Anna University Chennai, India. He is working as a senior lecturer in the department of Information Technology (e-mail: dhananjay@annauniv.edu).

Dr. Chellappan $\mathbf{C}$ is with Anna University, Chennai, India. He is working as a professor and head in the department of computer science \& engineering (e-mail: drcc@annauniv.edu).

Bala Jeevitha Vani B is with Anna University, Chennai, India. She has just completed her Master of Technology in Information Technology (e-mail: jee_vaani@yahoo.co.in).

Submitted $1^{\text {st }}$ April 2009. Published as resubmitted by the authors on 9 October 2009. 